\title{
MISCELÁNEA
}

\section{DEL LATÍN AL ESPAÑOL (*)}

Ramón Santiago

1. El libro es parte de una obra que pretende ofrecer una panorámica completa de la evolución histórica del español en sus aspectos fónico, morfológico y sintáctico. En este volumen se aborda el primero de ellos en su totalidad junto con el estudio de la morfología flexional, quedando para la continuación la descripción de la formación de palabras y la sintaxis. No es un manual más, mera refundición de los anteriores, porque el autor se ha propuesto colmar un vacío real que no puede por menos de haber lamentado cualquier estudioso de la lingüística histórica de nuestros días: la ausencia de una síntesis de lo mucho que se ha investigado ya en el esclarecimiento de problemas de interpretación en el desarrollo de las lenguas romances en su conjunto y también, específicamente, de la española. Su objetivo, pues, ha sido, según él mismo declara, tener en cuenta e integrar en una explicación coherente lo más importante de lo publicado hasta los primeros años de esta década ${ }^{1}$.

2. Consta el libro de cinco capítulos: "Sobre la naturaleza del cambio lingüístico" (págs. 1-69), "La lengua latina” (págs. 70-104), "Del latín arcaico al latín tardío" (págs. 105-170), "Del latín tardío al español antiguo" (págs. 171-315) y "Del español medieval al moderno" (págs. 316-368). Se completa el volumen con la bibliografía utilizada (págs. 369-389), una relación de palabras y morfemas estudiados (págs. 391-436) y un indice de temas (págs. 437-439).

* Reseña de Paul M. Lloyd, From Latin to Spanish. Vol. I: Historical Phonology and Morphology of the Spanish Language. Memoirs of the American Philosophical So ciety, vol. 173. Philadelphia, 1987, iii +439 págs.

1 Preface, pág. i. El profesor Lloyd manifiesta que tenía terminado el trabajo en 1981 y que en la redacción definitiva pudo aún incorporar algunas investigaciones más recientes. Efectivamente, hay en la bibliografía un buen número de títulos de 1982 y 1983, unos pocos de 1984 y 1985, y uno, al menos, de 1986. 
Dos novedades cabe destacar ante todo, en comparación con lo que suele ser habitual en los manuales y estudios de gramática histórica. La primera, el hecho de que se anteponga a la consideración específica de los procesos históricos un capítulo dedicado a la discusión de explicaciones antiguas y más modernas sobre el cambio lingüístico (tema nunca agotado, pero demasiado frecuentemente eludido por la disparidad de criterios y dificultad de soluciones), si bien reducido al que se estudia en este volumen: el fónico (págs. 1-55) y el morfológico (págs. 55-68). Otra, la ordenación del material, en los capítulos siguientes, en función de las sincronías o estados de lengua a que los procesos fonéticos y morfológicos dan lugar, en vez de ofrecer una descripción ininterrumpida de la evolución de sonidos o paradigmas desde el latín al castellano moderno. Este era ya el camino marcado por la fonología diacrónica, pero no era habitual proceder de manera semejante en morfología. En este libro se han incluido en las mismas secciones ambos tipos de cambios según las épocas y los sistemas, lo que es procedimiento lógico y sin duda deseable ${ }^{2}$.

3. No declara expresamente el Dr. Lloyd el método al que va a ajustarse la descripción e interpretación de los cambios a lo largo del libro, si bien su actitud teórico-metodológica queda suficientemente definida cuando al final del capítulo primero afirma: "We can expect that in the future the contribution of structuralism, and, more recently, the revealing investigation of the relationship between language change and social attitudes and social change will become part of the general linguistic knowledge of all students" 3 . Es, efectivamente, la metodología estructural la que constituye el armazón fundamental que vertebra el conjunto prestándole sólida coherencia expositiva, a la vez que se concede atención primordial a las circunstancias socioculturales de la comunidad lingüística en la que ocurre el cambio, actuando desde el convencimiento de que es imposible dar cuenta cabalmente de la evolución lingüística pura y simplemente en función de explicaciones "internas" «.

Ambas cosas están indudablemente justificadas.

2 Puede discutirse la oportunidad de colocación de algunas explicaciones: el imperfecto en -ie se alude en el capítulo del español medieval (pág. 300), pero se estudia en el de los resultados modernos (págs. 361-364); de modo semejante, la eliminación de las formas del posesivo to, so (pág. 353); la diptongación se trata dentro del capítulo del latín (págs. 117-130), mientras que la influencia de la yod, que la impide, sólo en el dedicado al español antiguo (págs. 193-199).

8 Cap. 1, Summary, pág. 69.

- Expresamente lo recuerda luego (por ejemplo, al comienzo del apartado The Constitution of the Castilian Dialect, cap. 4, pág. 172) y es una constante en toda la exposición. 
En primer lugar, en cuanto a su postura básicamente estructuralista: porque es la que también mayoritariamente subyace -0 , en todo caso, no repugna - a las aportaciones más interesantes a la lingüística histórica romance y particularmente a la del español, también en estos últimos años, al menos en el nivel fónico y morfológico, que es el que más se presta a esta metodología. De modo que el autor no ha tenido que forzar ni, menos aún, distorsionar las interpretaciones para adaptarlas al esquema fundamental de la exposición.

Probablemente no hubiera sobrado un espacio expresamente dedicado a esta cuestión de procedimiento, especialmente necesitada hoy de justificación -y aun clarificación - teórica y sobre la que se ha escrito ya quizá tanto como sobre la propia concepción del cambio linguístico, tema que no le es ajeno, desde luego. De todas formas, el autor no sólo ha evitado adoptar una postura metodológicamente radical, sino que se manifiesta expresamente contrario a cualquier dogmatismo y aboga por una actitud abierta, no ignorante de las aportaciones útiles del pasado, pero, a la par, capaz de asumir las innovaciones (una actitud suscribible, sin duda): "The progress of knowledge inevitably involves building upon past accomplishments as well as on news insights into the nature of language and its development" *.

En cuanto a la necesidad de prestar atención al factor social como ingrediente indispensable para entender y explicar, especialmente, el éxito (o el fracaso) de las innovaciones lingüísticas, hoy, a diferencia de lo que puede suceder en relación con el aspecto anterior, casi viene a resultar lugar común veinte años después del artículo "programático" de U. Weinreich, W. Labov y M. I. Herzog de 1968, más las posteriores publicaciones del propio Labov, que el autor tiene presentes de modo muy especial ${ }^{\circ}$. Pero ha sido objeto de atención preferente también y tema grato a la tradición que podemos decir genuinamente española en lingüística histórica e historia de la lengua desde, por lo menos, $\mathrm{R}$. Menéndez Pidal, oportunamente recordado aquí ${ }^{7}$, y nunca alejado de la preocupación intelectual de $\mathrm{Y}$. Malkiel ${ }^{\bullet}$, cuyas ideas se tienen muy en cuenta en este libro, y del que el profesor I loyd se siente particular deudor ${ }^{\circ}$.

- Cap. 1, Summary, pág. 69.

- Cf., sobre todo, cap. 1 , págs. 13 y sigs.

7 En una ocasión llega a apostillar la famosa frase laboviana "The origin of a change is its propagation or acceptance by others", con la observación de que "Labov's point of view echoes Menéndez Pidal" (pág. 55, nota 83).

- No puede olvidarse que fue Malkiel uno de los editores de Directions for Historical Linguistics, donde se publicó el trabajo de Weinreich, Labov y Herzog, "a crucially important monograph". en palabras del propio Malkiel (cf. "Necrology-Uriel Weinreich, Jakob Jud's Last Sudent", en RomPh, XXII, 1 (1968), pág. 131).

- Cf. Preface, pág. ii, y Bibliografía, págs. 379-380. Destacan de modo especial la 
Ésta es, quizá, la parte más satisfactoriamente desarrollada en el planteamiento del cambio lingüístico - fonético, más exactamente- y de sus formulaciones, que se discuten y se valoran en el capitulo primero en toda su complejidad con sugerencias sumamente pertinentes ${ }^{10}$.

4. Por lo que se refiere al contenido que es objeto específico del libro, se debe decir sin vacilación que resultará una ayuda inestimable y de consulta obligada para cuantos se esfuerzan por estar al día y buscan una información ponderada, fiable y actualizada del estado de la cuestión en torno a los principales problemas que atañen a la evolución fonética y morfológica (flexional) del español. Es más, el autor no se ha limitado a trasladar opiniones acumulativamente, sino que las ha valorado críticamente con agudeza, poniendo en práctica de alguna manera lo que él mismo señala en el capítulo primero como un desideratum: la atención a la jerarquización de las causas del cambio lingüístico ${ }^{11}$. Ni faltan tampoco sus propias aportaciones y reelaboraciones personales: una de las más relevantes, sin duda, es la de la estructura silábica latina y romance, tema, por lo demás, bastante descuidado en nuestra bibliografía, a pesar de su trascendencia histórica a través de procesos diversos ${ }^{12}$.

de la multicausalidad en el cambio (véase ya, cap. 1, pág. 69) y la de la influencia de factores morfológicos en el cambio fonético (por ejemplo, págs. 316-320, sobre la reducción de los diptongos ie/ue, a pesar de las precauciones tomadas en el cap. 1, pág. 51).

10 Una pregunta nada intrascendente queda en el aire ya desde ahora, al tratar de la regularidad del cambio fonético y las "leyes" fonéticas: "How many examples do we need to establish a general law, and, conversely, how many exceptions to a putative law are required to invalidate such a law?" (La formulación expresa, retomando las ideas de la introducción, la hace el autor mucho más adelante, a propósito de cambios concretos en la historia del español : cap. 4, pág. 181).

Otros aspectos más tradicionalmente tratados pueden resultar menos interesantes. Se describen, por ejemplo, con cierto detenimiento, en la discusión sobre el cambio lingüístico, los "tipos de cambios de sonidos" (= fonéticos : asimilación, disimilación, metátesis, síncopa, apócope, aféresis, epéntesis, págs. 6-8; más adelante, pág. 28 , se habla también de los "cambios esporádicos": especialmente la metátesis, varios tipos de epéntesis, disimilación, anaptixis y paragoge), pero no se da cuenta, paralelamente, de una tipología de cambios "fonológicos", continuamente aludidos y utilizados a lo largo del libro. Y a propósito de los cambios fonéticos, se podría discutir, de paso, la pertinencia de la relación y descripción mencionadas, salvo que se tratara de superar los límites tradicionales para abordar también los "cambios de sonidos" que no recogen esos nombres (¿cuál de esos tipos es la diptongación de $e / o$ tónicas y abiertas, la consonantización de [w], la palatalización de [nn] o [11] geminadas, etc., etc.?).

11 Cap. 1, Summary, pág. 69.

12 Ya había llamado la atención sobre el particular D. Catalán ("En torno a la estructura silábica del español de ayer y del español de mañana", Sprache und Geschichte. Festschrift für Harri Meier sum 65. Geburtstag, München, 1971, págs. 77-110). E1 
Claro está que en un trabajo de la envergadura del emprendido por el Dr. Lloyd, más llevadero, a estas alturas, como labor de equipo que en solitario -y siendo así, tanto más admirable-, no resulta difícil de entender que la lectura sugiera nuevas preguntas o que puedan encontrarse aspectos menos satisfactoria o convincentemente tratados. Al fin y al cabo, como él mismo dice respecto del desacuerdo de los lingüistas en torno a la explicación de los cambios (y puede ello trasladarse a otras muchas discusiones): "much will inevitably depend on the investigator's viewpoint, his goals, and the level on which he chooses to work" 18. Las que señalo a continuación son algunas pequeñas cuestiones que quizá merezcan tomarse en consideración en ediciones futuras.

4.1. Al aspecto gráfico se dedica alguna atención, especialmente a propósito de los cambios del sistema medieval que dan lugar al moderno. Como indicio gráfico de indistinción sorda-sonora, se aducen testimonios tomados de los Documentos Lingǘsticos de Castilla de entre 1100 y 1288, todos, menos siete, anteriores a 1240, que mostrarian que "the spelling 'errors' that are found appear earlier [que en otras partes] and in greater numbers in documents from Castile" ${ }^{14}$. Se trata de 11 imperfectos de subjuntivo, más las formas uasalos y pasare, siempre con $-s$ - simple, en lugar de la doble de la grafía estandarizada, y 23 palabras ${ }^{16}$ con $-c-0-c_{-}$, en lugar de $-z-$, la mayoría de ellas formas verbales de los derivados de lat. facere, dicere, iacere. Sin entrar ahora a discutir formalmente lo que fuera la extensión del ensordecimiento o indistinción sorda-sonora en la Castilla de esa época, creo que es sumamente cuestionable, al menos, justificar una argumentación sobre este tipo de datos, extraídos de documentos anteriores, casi todos, a la "normalización" gráfica de mediados del siglo xirr. En esos documentos pueden encontrarse a la vez otros varios tipos de "errores", que, tomados como tales, deben hacer dudar hasta de que el sistema fonológico subyacente haya tenido algo que ver con el que comúnmente se admite. Pero es que, además, tras la "normalización" gráfica pueden seguir viéndose hechos parecidos a los reseñados anteriormente. Por ejemplo, en la primera parte de la Primera

\footnotetext{
profesor Lloyd discrepa del supuesto de que en cierto momento de la evolución del castellano medieval llegara a predominar la sílaba trabada por efecto de la acción sucesiva de síncopa y apócope (cuestión que no cuenta, desgraciadamente, con estudios similares a los efectuados por Navarro Tomás para otras épocas de la lengua), pero su planteamiento viene a corroborar que ciertas evoluciones fonéticas se conforman con pautas específicas de estructura silábica.

18 Cap. 1, pág. 68.

14 Cap. 5 , pág. 329 y nota 10.

16 Una de ellas $(v a c ̧ a)$ del doc. n. ${ }^{\circ} 64$, lín. 27, de Frías (año 1278), está confundida: es yaçe (por cierto, con $z$ visigótica, más que cedilla).
} 
Crónica General (es decir, la redactada en el reinado del propio Alfonso X) se puede leer: asaz, uasallage, espesas (del lat. spissas), (as)sesegó, sesegados, assesegadamientre, detardase, prisiesen, etc.; y, por el contrario, cassa, guissa, guissassen, guissaronse, pusso, pussieron, quissiessen, pessar, etc., etc. Y considerando esta obra en su conjunto (las dos partes de la Crónica), hay más de 100 imperfectos de subjuntivo, más de 100 formas derivadas de lat. passare, y al menos una veintena de veces la que procede de lat. uassallus, unos y otras con $-s$ - simple ${ }^{16}$. Creo, pues, que hay que examinar previamente cuándo y por qué la grafía puede o debe tomarse como indicio de indistinción (o de distinción) fonética. Entre negarle toda validez por sí sola ${ }^{17} \mathrm{y}$ aceptar cualquier hecho gráfico como confusión fonética real hay demasiada distancia. Sesenta páginas antes el autor había adoptado una actitud más precavida acerca de las grafías antiguas: "it may be that in the earliest period we simply do not have the written evidence most necessary for a resolution of our difficulties" ${ }^{18}$. Se trata ahí de las grafías $\varsigma$ y $z$. Pero ¿acaso ocurre eso solamente con ellas?

A caballo, también, entre lo puramente gráfico y lo estrictamente fonéticofonológico está el asunto nada irrelevante de la utilización de $y$ y el estatus del sonido que representa en el sistema medieval. Son sumamente razonables algunos de los tratamientos que se hacen de esta articulación conflictiva a lo largo del libro, caso, por ejemplo, del planteamiento sobre la alternancia $y$ - / $j$ - en posición inicial ${ }^{18}$. Pero, aparte definirla, al menos en una ocasión, como "a palatal obstruent $[y]$ " ${ }^{20}$, no se concede mayor atención a ese pro-

18 Incluso la única vez que parece documentarse la palabra derivada de lat. puteus en la Primera Crónica, está escrita con $\varsigma$ : poços (311 b 8). Las palabras aducidas arriba pueden encontrarse, al menos, en los lugares siguientes de la edición de Menéndez Pidal: 43 a 5 y $8 ; 273$ a $32 ; 290$ a $8 ; 30$ b 39 y 281 a $6 ; 14$ a $3 ; 24$ a $44 ; 285$ a $5 ; 244$ b 22 ; 269 a $15 ; 243$ b $30 ; 243$ b $33 ; 10$ b $31 ; 31$ b $12 ; 31$ b $11 ; 243$ b 18 y 26 . Datos cuantitativos de estas y otras formas pueden verse en el "Index Alphabétique des formes de la 'Primera Crónica General" de Jean Roudil (Cahiers de Linguistique Hispanique Médiévale, n. 4 bis, 1979) y, naturalmente, en las Concordancias editadas por el Seminario de Estudios Medievales de la Universidad de Wisconsin (Madison, 1978).

17 Cf. A. Alonso, De la pronunciación medieval a la moderna en español, vol. $2^{\circ}$, Madrid, 1969, pág. 11.

18 Pág. 263. A pesar de esto, cuatro páginas más adelante (267, nota 97) se argumenta también con la - $\varsigma$ final (tipo Guterrę -i. e. Gutiérrez-) en documentos de 1197 y 1226.

10 Concretamente, págs. 248-252. Ahora debe tenerse en cuenta, además: J. A. Pascual, "La lengua del Registro Antiguo. Algunos problemas gráficos y fonéticos" (en Documentos y estudios para la historia del occidente peninsular durante la Edad Media. Propiedades del cabildo segoviano. Sistemas de cultivo y modos de explotación de la tierra a fines del siglo XIII. Bajo la dirección de José-Luis Martín. Salamanca, 1981, págs. 169-184), especialmente págs. 169-177.

20 Como resultado de la palatalización de $d$ y $g$ : pág. 194. 
blema, a diferencia de la prestada a otras fonologizaciones. Dado el desacuerdo sobre este particular en la bibliografía especializada ${ }^{21}$ no habría estado nada de más dedicarle algún espacio. Algo semejante puede decirse respecto de lo que representa $g$ (ante vocal palatal) o $i, j$, fonema tradicionalmente ambiguo en las descripciones históricas ${ }^{22}$.

Otras cuestiones gráficas afectan más que nada a la presentación ${ }^{23}$.

4.2. La descripción y explicación del nivel fonético, que son, sin duda, las más completas y las de mayor calidad técnica de cuantas se han intentado hasta la fecha, pueden, a la vez, sugerir algunos otros pequeños temas para la discusión.

El efecto de la yod sobre vocales y consonantes, además de ser central en fonética histórica romance, es también un problema tradicional de explicación y, en una parte, al menos, de sistematización. Así, concretamente, todos los intentos de clasificar las inflexiones vocálicas ensayados tropiezan con dificultades similares, más a la vista cuanto más aumenta el volumen de datos, mientras que en la mayor o menor apariencia de regularidad pueden influir no poco los ejemplos elegidos o excluidos. El esfuerzo del autor, en este caso, pretendiendo hacer una presentación "más fácil" 24 es muy estimable y no merece mayor objeción por el hecho de que no "justifique" nás que otras ciertos comportamientos ${ }^{25}$. Sí parece, sin embargo, que puede añadirse alguna irregularidad más de la prevista con otros ejemplos distintos de los propuestos ${ }^{26}$ y que puede caber algún otro reparo de formulación en puntos concretos ${ }^{27}$.

21 Además de E. Alarcos (Fonología, 4. ${ }^{\mathrm{a}}$ ed., 1965, págs. 265 y 277) y aun A. Martinet (Economia, 1974, pág. 461), cf. C.-P. Otero, Evolución y revolución en romance, Barcelona, vol. 1. ${ }^{\circ}, 1971$, págs. 105-106, y vol. 2. ${ }^{\circ}, 1976$, págs. 104-107; M. Ariza, "Sobre la palatal sonora no rehilada en español antiguo", en Estudios ofrecidos a E. Alarcos Llorach 2., Oviedo, 1978, págs. 1-22, y "Sobre las palatales sonoras en español antiguo", en Introducción plural a la Gramática Histórica (Francisco Marcos Marín, coord.), Madrid, 1983, págs. 31-51.

22 Cf., por ejemplo, E. Alarcos, Fonología, págs. 265 y sigs. El profesor Lloyd utiliza constantemente el símbolo ž.

28 Así, el hecho de que, en la misma página y lista de ejemplos, se escriba, sin que se advierta el motivo, unas veces $-n b$ - y otras -mb- (canbra, cogonbro, onbro, pero membrar y tembrar, pág. 203), unas veces -sc- y otras -c-(ofrescer, parescer, padescer, pero parecer: pág. 289; también descir, pág. 291).

24 Cap. 4, pág. 193. El autor parte del trabajo de J. R. Craddock, "The contextual varieties of yod. An attempt at systematization", en A festschrift for Jacob Ornstein. Studies in general linguistics and sociolinguistics, Rowley, 1980, págs. 61-68.

25 Como el de la vocal tónica de strictu (pág. 196) frente a tructa (pág. 197) o el de la de sedeat (pág. 195) frente a uideat (pág. 197), etc., dados los mismos entornos.

26 Tal sería el caso de lat. nauigiu > navio (con $i$ y no la $\dot{e}$ de los de su grupo, pág. 196) o lat. rubeu > rovo (con ó y no la ú del resto, pág. 197), etc. 
En lo que atañe a las consonantes, y sin salir del ámbito del influjo de la yod, hubiera sido de interés que se razonara el supuesto de la igualación absoluta de las secuencias lat. -gn- y $-m n$-: ambas habrían dado -nn-, como la geminada originaria $y$, finalmente, $-\underline{n-28}$; pero, siendo así, no se ve por qué unas - $n$ - inflexionan (así en el caso de lat. pugnu $>$ puño ${ }^{29}$ ) y otras no (la de lat. autumnu o cunnu). También podría haberse indicado por qué se opta por el resultado intermedio / $1 /$ a partir de $-l j-/-i l$-, antes de dar $/ \check{z} /$, paso no indefendible teóricamente, desde luego, pero que ha sido puesto en tela de juicio expresamente por diferentes autores y con argumentos igualmente varios ${ }^{30}$. Ni hubiera sido inútil dedicar alguna atención a precisar o aclarar ciertas evoluciones de grupos consonánticos, apartado tradicionalmente deficiente en los manuales y poco frecuentado bibliográficamente: por ejemplo, la justificación fonética de la doble vocalización, en $-i$ - o en $-\mu-$, de la $-l$ en posición implosiva (lat. saltu, multu) ${ }^{\mathbf{3 1}}$; o la de la posibilidad de vocalización de una velar implosiva tras consonante (como en el tipo conchula $>$ concha o cingulu $>$ ceño) ${ }^{32}$; o señalar en qué consiste el "reforzamiento de la palatal" [1] que transforma lat. singulos en sendos ${ }^{38}$.

27 No deja de parecer cierta contradicción el hecho de que se presente a $/ \mathrm{l} / \mathrm{y} / \mathrm{n} /$, resultados de la palatalización de $l$ y $n$, como semejantes ("to some extent") en sus efectos a los de $t$ y $k$ palatalizadas por yod semiconsonante ("were simple consonants and had no further effect on their surroundings", pág. 193), cuando en la página siguiente se advierte que actúan como los resultados de la palatalización de $d$ y $g$ : "we must include as well the palatal consonants $/ \mathrm{l} /$ and $/ \mathrm{n} / \ldots$ Although the yod was absorbed by the consonant, new palatal consonants acted much like those ..." (pág. 194).

Tampoco resulta mucho más satisfactorio observar que sedeat $>$ sea figura como ejemplo de contextos "in which the yod is preserved" (y por lo tanto hay inflexión, pág. 195) junto a soberbia, nervio, premio y grey, mientras que uideat $>$ vea lo es de contextos "were the yod disappeared" (y por lo tanto se mantiene la vocal sin modificación, págs. 196-197), lo mismo que cervesa, estrecho, correa, deseo, etc.

Podría matizarse también, en cuanto a las vocales átonas, la afirmación de que la yod inflexiona "invariably" las pretónicas (pág. 199); y no encaja demasiado bien en este apartado, que lleva por título "The yod and the Petronic Vowels", lo dicho primeramente acerca de $/ \mathrm{l} / \mathrm{y} / \mathrm{m} /$ desde el momento en que aqui figuran, entre otros ejemplos de esta inflexión, palabras como muliere o cognatu. ( $\mathrm{Y}$ acerca de -gn-, por otra parte, véase a continuación, en el texto.)

23 Págs. 140 y 244.

29 Pág. 197.

so Págs. 134 y sigs., y especialmente 244. Cf. a este respecto, el resumen de la extensa nota 10 del $\S 41$ de la Historia de la Lengua Española de R. Lapesa (págs. 169170 de la ed. de 1980, que es la que se incluye en la bibliografía).

31 Cap. 3, pág. 190, y cap. 4, pág. 254.

82 Cap. 4, págs. 254-255. Para el primer tipo de cambio supone: "posconsonantal $/ \mathrm{l} /$ derived from $-\mathrm{jl}-<-\mathrm{g}^{\prime} 1$. gives $/ \check{c} /$ as does the $/ \mathrm{H} /</-\mathrm{pl}-,-\mathrm{fl}-/$ as we saw 
También podría haberse tenido en cuenta el resultado sonoro (grafía -z-) en el caso del grupo -cons. $+d j$-, que también existe, contra lo que se deduce a este respecto ${ }^{34}$; o los dobles resultados $(-\varsigma / z-)$ de $-d j$ - en posición intervocálica (bayo/baço, raya/raça) ${ }^{35}$. Así mismo, al tratar el resultado típicamente castellano, frente al de otros dialectos, de -sk + yod- ${ }^{86}$, deberá añadirse que en igual situación están $-s t+$ yod- $(u \varsigma o)$ y $-s k+$ vocal palatal(crecer) ${ }^{87}$. Y, para cerrar el capítulo de las palatalizaciones, se echa en falta la mención de una de ellas, por más que se produjera de modo escaso o aun esporádicamente: la de -ssj-(tipo quexar(se) o baxar), de la que no parece haber una sola cita.

Algunas pocas -y pequeñas - ausencias más podrían tomarse en consideración.

Por ejemplo, la de la alternancia $g-/ k$ - en posición inicial, sobre la que hay investigaciones conocidas ${ }^{38}$. O la de la apócope de $-o$ al lado de la de $-e$, de cuyo proceso se hace, ciertamente, un excelente resumen ${ }^{39}$. Además, por formularse desde los finales consonánticos modernos, no se menciona la po-

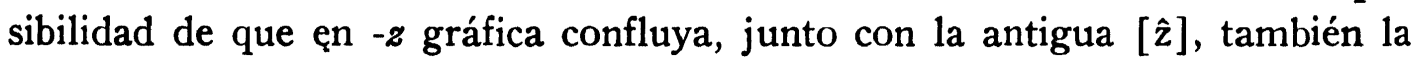

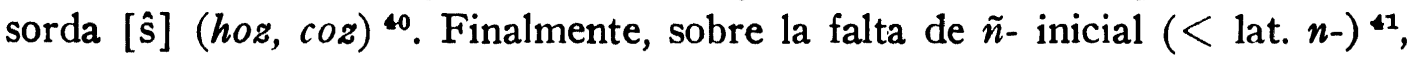

previously". (¿Con qué vocal formaría diptongo la yod?) Este último caso se trata, sin descripción del proceso, en la pág. 228.

Queda sin especificar qué consonantes se mantienen o desaparecen (concha frente a macho).

En el caso de cingulu se dice: "the palatal has simply been absorbed by the $/ \mathbf{n} /$, producing $/ \mathfrak{b} /$ ".

88 Cap. 4, pág. 256, nota 85 .

s4 Cap. 4, pág. 255. Acerca de granza, cf. DCECH s. v. Además, naturalmente: almucrzo. Igualmente habría que tratar el caso de gozo.

85 De -dj-se habla en las págs. 247-252, y sólo de la solución y 0 y-/j-.

- Cap. 4, págs. 259 y 274.

87 Cf. R. Menéndez Pidal, Orígenes, § 57.3; Lapesa, Historia, §§ 44.2 y 46.2 (págs. 180 y 186).

88 Cf. G. Salvador, "Neutralización G-/K- en Español", en Actas del XI Congreso Internacional de Lingüística y Filología Románica, Madrid, 1969, págs. 1.739-1.752 (ahora, con una "Apostilla de 1985 con algunas consideraciones sobre el rasgo de sonoridad", en Estudios dialectológicos, Madrid, 1985, págs. 152-167); F. González Ollé, "La sonorización de las consonantes sordas iniciales en vascuence y en romance y la neutralización de k-/g- en español", $A O$, XXII (1972), 253-274.

89 Cap. 4, págs. 207-212.

10 Sólo se da ejemplo de sonora: pace > paz. Lo mismo más adelante, cuando se plantea la pregunta de si -z puede representar en realidad una articulación sorda o ensordecida, a propósito de la palabra luz (pág. 259). Sería pertinente aquí: A. Alonso, "La '-z' final", De la pronunciación, vol. 2. ${ }^{\circ}$ págs. 158-172.

41 Cap. 4, pág. 247 y nota 78. 
no parece resultar menos atendible la observación de Menéndez Pidal ${ }^{\star 2}$ que la de Martinet sobre la supuesta influencia vasca.

Dentro aún de este apartado, sería posible mejorar formulaciones menos felices o evitar pequeñas inexactitudes, sin duda involuntarias unas y otras, que se habrán deslizado contra la voluntad diligente del autor ${ }^{43}$. Y aunque no se trate de ninguna de esas cosas, no dejará de resultar extraña para nuestros hábitos la identificación, sin más advertencia, de la $r$ vibrante múltiple con una geminada 4 .

4.3. El nivel morfológico ocupa, por razones obvias, mucho menos espacio (103 páginas en total, frente a las 258 del fónico), incluso ya desde el capítulo primero, en que la discusión sobre la naturaleza del cambio morfológico en general queda reducida a la consideración de la analogía (12 páginas) ${ }^{45}$.

Dice el profesor Lloyd en la introducción ${ }^{\star 6}$ que la reciente publicación de la Morfología histórica del español, de M. Alvar y B. Pottier, ha reducido la utilidad de estas páginas de su libro, si bien algunos aspectos del estudio pueden seguir teniéndola. Se puede estar de acuerdo en ambas su-

12 "Dos problemas iniciales relativos a los romances hispánicos", en $E L H$, I (Madrid, 1960), págs. cxxviii-cxxix.

4 Así, la inclusión de lat. balineae > balneae como ejemplo de síncopa de vocales átonas, en latín (pág. 113); la distribución de los grupos consonánticos latinos de la página 139 , que se solapan entre sí (grupos "con fricativa", entre los que se pone $p s, \mathbf{y}$ grupos "con oclusiva en el primer miembro", donde figura $k s$ : ambos pertenecen, en realidad, a los dos grupos; lo diferente es el resultado, no el antecedente); la formulación de la pág. 205 "the coming into contact of $/ \mathrm{p} /$ or $/ \mathrm{b} /$ plus $/ \mathrm{t} /$ produced the groups /bd/", cuando en todos los casos ha ocurrido la sonorización previamente (cabdal, cabdiello, recabdar, bebdo, etc.); o la de la pág. 232 "Only when / kw/ appeared before a tonic /a/ did the semiconsonant remain", cuando figuran a continuación, con otros ejemplos, las palabras antigua, agua y yegua; el hecho de que se diga que la palabra océano se pronuncia hoy "most frequently" con el acento en la $a$ (pág. 320). Finalmente, sobre la fecha que se da de las Glosas Emilianenses, en pág. 178 (siglo x), cf. M. C. Díaz y Díaz, Las primeras glosas hispánicas, Barcelona, 1978, pág. 30 ("siglo xI bastante entrado)"; y sobre la del Manual de escribientes de A. Torquemada, en la pág. 343 (1574), cf. la introducción de M. ${ }^{\mathrm{a}}$ Josefa C. de Zamora y A. Zamora Vicente en su edición (Madrid, 1970), págs. 12 y 13.

14 En la pág. 243 se dice que en cuanto a la $r$ geminada latina "no change at all occurred and thus the geminate has been preserved until today". Confróntese E. Alarcos, Fonología, pág. 248.

40 No es, por lo demás, procedimiento inhabitual. Así lo hace también Hans Henrich Hock en sus recientes Principles of Historical Linguistics, Trends in Linguistics. Studies and Monographs, 34, Berlin. New York. Amsterdam, 1986 (caps. 9-11).

¿ Preface, pág. i. 
posiciones y añadir que, respecto de la segunda, destaca, sin duda alguna, el tratamiento del verbo.

La sección de morfología nominal es la más simple desde el momento en que se excluyen de este volumen (hasta donde se puede) las funciones y significados gramaticales. Por ello vienen a resultar forzosamente esquemáticas la exposición y las conclusiones del proceso de eliminación de la declinación latina ${ }^{\star 7}$. El de la transformación del género, del que existe un considerable material acumulado, por lo menos desde los conocidos trabajos de A. Rosenblat y otros después, tampoco va más allá de su fase inicial, en los capítulos dedicados al sistema latino ${ }^{48}$, sin volverse a mencionar sino alguna vez, de paso ${ }^{4 \theta}$, más adelante. $\mathrm{Y}$ del número gramatical y sus particularidades en relación con la forma de los nombres no se dice nada específico.

En lo que respecta al sistema verbal, es ésta con mucho la parte más amplia, la desarrollada con más recursos y más en consonancia con el alto nivel de conjunto de la descripción fonética, aunque haya de reducirse fundamentalmente al estudio de las formas simples. El de los tiempos compuestos es otro de los puntos débiles tradicionales, especialmente en lo que se refiere a la interpretación del proceso de reestructuración del sistema temporalaspectual en su totalidad, tema que, lógicamente, el autor habrá reservado con cuantos puedan echarse aquí en falta, para el segundo volumen ${ }^{\text {*0 }}$.

Ciertamente hay (sigue habiendo) aspectos sumamente problemáticos ${ }^{\circ 1}$,

47 Cap. 2, págs. 152-153. No se menciona en toda esta evolución (ni siquiera en la relación general de los casos latinos) el vocativo, aunque como forma diferenciada sólo existiera en el singular de la segunda declinación y aun así, con la competencia siempre del nominativo. La primera referencia, si no me equivoco, de este caso está en el apartado de los llamados "restos fonéticos" de los casos, dentro del capítulo siguiente, pág. 276. (Falta, por cierto, la cita de esta cuestión en el "índice de materias", págs. 437-438).

Por otra parte, aún en este capítulo de los "restos fonéticos" habrá que excluir virtos como herencia de un neutro singular de la tercera declinación (pág. 275) y por lo menos discutir que maestre y pómez procedan de los nominativos singulares magister y pumex (pág. 276). (Cf. sobre maestre DCECH s. v. maestro; sobre pómez, M. Alvar y B. Pottier, Morfología, pág. 66.) Además, en la pág. 277, hay que añadir Santiuste al Santiyuste que ahí se cita (como procedente del lat. Sancti Iusti) y sustituir Romanci por Romanici.

48 Específicamente, cap. 3, págs. 154-155 y 156-157.

49 Por ejemplo, cap. 4, pág. 280.

so De la forma latina - no del origen- de los tiempos compuestos se trata en las págs. 169-170 del cap. 3 .

*1 Por ejemplo, casi todo el desarrollo de los perfectos de la segunda conjugación, especialmente las págs. 301-303 del cap. 4.

Mucho más localizado como problema pero tradicionalmente recalcitrante está la cuestión de la consonante sorda o sonora (sope, cope / yogue, plogue), pág. 304, para- 
pero globalmente es una muy cuidada síntesis de lo que hoy comúnmente se transmite y se discute sobre la morfología histórica del verbo español y, por supuesto, de lo que nos falta por saber.

5. En la bibliografía ${ }^{52}$ se consignan únicamente los trabajos citados ${ }^{58}$ a lo largo de la exposición. Está claro que si se opta por dar sólo una selección siempre se ofrece la posibilidad de que se pregunte el lector por las ausencias. Probablemente, aunque no fueran tales, no hubiera sido este libro sustancialmente diferente de lo que es. Realmente, reunir una bibliografía exhaustiva sobre un objeto de investigación tan amplio, de $\tan$ constante producción editorial y de no menos larga tradición como éste, ni es sencillo ni, en este caso, tenía por qué proponérselo el autor en absoluto, por más que a estas alturas va siendo ya una necesidad, y la publicación de un trabajo como el que nos ocupa no deja de recordarla. Además, la bibliografía reunida por el Dr. Lloyd es, seguramente, la más completa y más al día de las disponibles hasta la fecha sobre esta parte de la evolución histórica del español.

6. Nada de lo que antecede, pues -pequeñas cuestiones, discutibles las más-, debe impedir reconocer el mérito objetivo de este trabajo. El profesor Lloyd ha abordado con decisión una tarea necesaria y, también, sumamente difícil. $\mathrm{Ha}$ procedido con claridad de razonamiento, con ponderación $\mathrm{y}$ equilibrio, $\mathrm{y}$ ha conseguido ofrecer un panorama actualizado y razonablemente crítico de las principales cuestiones de la evolución fonética y morfológica del español.

Una presentación e impresión esmeradas ${ }^{\text {t }}$ hacen cómoda, además, la

lelamente a los casos de yod (sepa, quepa), pág. 298, aunque no se tiene en cuenta aquí plega.

152 References, págs. 369-389.

58 Alguno se ha pasado por alto: por ejemplo, el de E. Alarcos sobre los efectos de la yod en la vocal tónica, citado en la pág. 194, nota 18; o el de Y. Malkiel sobre el imperfecto de indicativo, citado en la pág. 36. También, en un caso al menos, se da en el texto una referencia a edición diferente de la que figura en la bibliografía: tal es la de Lapesa (1959) 177, en la pág. 327, que corresponde a la Historia de la Lengua Española. En la edición de la bibliografía (1980), será la pág. 259.

64 Soy muy pocas las erratas y de escasa relevancia, en general. Alguna se ha señalado ya. Añado sólo una que tal vez pueda perturbar la comprensión: en la pág. 333 , nota 17 , se atribuye a A. Alonso el convencimiento de que "the $\epsilon$ became deaffricated first", cuando evidentemente debe tratarse de $z$ (cf. De la pronunciación, vol. I, 1969, 2. a edición, págs. 309, 314, etc.).

Finalmente, entre las págs. 309-313, notas $117-119$, hay confusión de números de notas en el texto y de las propias notas a pie de página; y en las págs. 206, 265, 279 y 309 falta la cifra de página que remite a asuntos tratados (respectivamente : 114, 242243, 192 y 292), quedando en su lugar la indicación de las pruebas de imprenta (000). 
lectura, y los índices de palabras y morfemas y de materias facilitan extraordinariamente la consulta.

Evidentemente (se puede decir que afortunadamente, tal vez) no todo está resuelto y quedan aún bastantes caminos que frecuentar. Pero también por esto, por sugerir o dejar descubrir de qué estamos más necesitados, es uno de los libros más útiles en la bibliografía de la lingüística histórica de los últimos años. Hacía falta una obra así. Sólo cabe desear que la aparición del segundo volumen no se haga esperar mucho tiempo y que sea pronto traducida al español para su mayor difusión en el mundo hispanohablante. 